\title{
Current state of navigation in spine surgery
}

\author{
Nathaniel Rawicki ${ }^{1}$, James E. Dowdell ${ }^{2}$, Harvinder S. Sandhu ${ }^{2}$ \\ ${ }^{1}$ Department of Orthopedics, Westchester Medical Center, Valhalla, NY, USA; ${ }^{2}$ Department of Spine, Hospital for Special Surgery, New York, NY, USA \\ Contributions: (I) Conception and design: JE Dowdell; (II) Administrative support: HS Sandhu; (III) Provision of study materials or patients: JE \\ Dowdell, N Rawicki; (IV) Collection and assembly of data: JE Dowdell, N Rawicki; (V) Data analysis and interpretation: JE Dowdell, N Rawicki; (VI) \\ Manuscript writing: All authors; (VII) Final approval of manuscript: All authors. \\ Correspondence to: James E. Dowdell. Department of Spine, Hospital for Special Surgery. 535 E 70th St, New York, NY 10021, USA. \\ Email: dowdellj@hss.edu.
}

\begin{abstract}
The use of navigation has become more prevalent in spine surgery. The multitude of available platforms, as well as increased availability of navigation systems, have led to increased use worldwide. Specific subsets of spine surgeons have incorporated this new technology in their practices, including minimally invasive spine (MIS) spine surgeons, neurosurgeons, and high-volume surgeons. Improved accuracy with the use of navigation has been demonstrated and its use has proven to be a safe alternative to fluoroscopic guided procedures. Navigation use allows the limitation of radiation exposure to the surgeon during common spine procedures, which over the course of a surgeon's lifetime may offer significant health benefits. Navigation has also been beneficial in tumor resection and MIS surgery, where traditional anatomic landmarks are missing or in the case of MIS not visible. As cost effectiveness improves, the use of navigation is likely to continue to expand. Navigation will also continue to expand with further innovation such as coupling the use of navigation with robotics and improving tools to enhance the end user experience.
\end{abstract}

Keywords: Navigation; computer assisted navigation; minimally invasive spine surgery (MIS surgery); spine surgery; image guided

Submitted Feb 05, 2020. Accepted for publication Jun 16, 2020.

doi: 10.21037/atm-20-1335

View this article at: http://dx.doi.org/10.21037/atm-20-1335

\section{Introduction}

Technological advances in spine surgery have been immense, with changes in: operative technique, approaches, biologics, implants, and imaging. With the complexity of the three-dimensional anatomy of the spine and the sensitive nature of the neurovascular structures involved, innovation has optimized safety and efficiency during spine surgery. One change within surgical treatment of the spine was the onset of widespread use of Computer Assisted Navigation (CAN), or colloquially referred to as navigation, in an effort to maximize efficiency and limit complications associated with surgical trauma. To combat the relative inaccuracy of traditional methods of pedicle screw placement and the high stakes of screw misplacement, CAN was introduced as a means to improve accuracy (1). As minimally invasive spine (MIS) surgery became more prevalent, CAN was used to limit radiation exposure to both the surgeon and patient (2). The current demands of spine surgery make the use of navigation something that must be considered given the potential to improve accuracy. Current advances in imaging technology and accuracy have made the use of navigation more prevalent. The purpose of this manuscript is to provide the background behind the use of computer assisted navigation, the proposed benefits and limitations, and the future of its use within spine surgery.

\section{History/examples of computer assisted navigation}

The first case report discussing the successful use of computer assisted imaging systems was in 1995, showing successful integration of real time information with threedimensional anatomy for successful placement of open 


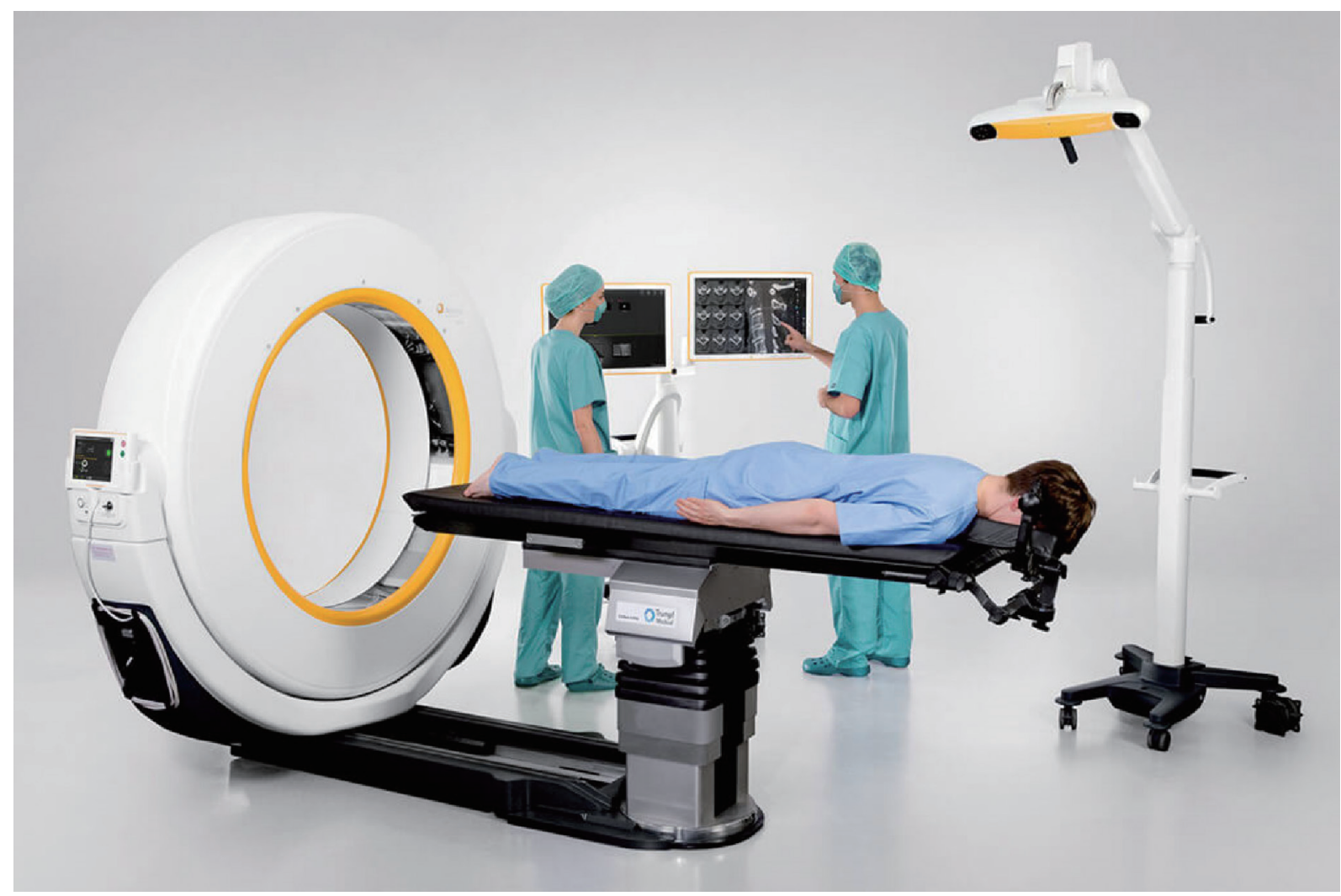

Figure 1 Airo mobile intraoperative CT/Brainlab.

lumbar pedicle screws (1). After the first case reports of successful navigation use within spine surgery, further research began regarding its accuracy, cost effectiveness, and safety $(3,4)$. Multiple medical technology companies began to address each of those problems individually, leading to development of multiple concurrent systems of navigation currently in use within spine surgery.

There are many systems currently available for CAN. In 2017, Overley et al. summarized many of the current platforms, including: Airo Mobile Intraoperative computer tomography (CT)-based Spinal Navigation $\left(\right.$ Brainlab $^{\odot}$, Feldkirchen, Germany), Stryker Spinal Navigation with SpineMask $^{\odot}$ Tracker and SpineMap Software (Stryker ${ }^{\odot}$, Kalamazoo, Michigan), Stealth Station Spine Surgery Imaging and Surgical Navigation with O-arm (Medtronic ${ }^{\odot}$, Minneapolis, Minnesota), and Ziehm Vision FD Vario 3-D with NaviPort integration (Ziehm Imaging ${ }^{\odot}$, Orlando, Florida) (5).

The Airo Mobile Intraoperative CT-based CAN platform was one of the early pioneers of the navigation platforms. This system was mobile with a large diameter circular scanner which is attached to the operating table and allows for full 360-degree scanning (Figure 1). The instruments used in this system have 3 attached reference points that are recognized by the system's scanning stereotactic camera, calibrated prior to intra-operative scanning, and then coupled with an anatomic reference clamp attached to a spinous process (after exposure) or to the iliac crest via pins in percutaneous cases. OR staff may exit the room during scanning to reduce radiation exposure. The obtained image is automatically registered to the attached software, generating a real-time three-dimensional map for instrumentation guidance (5).

The Stealth Station with O-arm (Medtronic) and the ZiehmVision FD Vario 3-D are two very similar systems in terms of technology. The O-arm is a 360-degree scanner, but opens at 90 degrees for mobilization around the patient. The Ziehm Vision FD Vario 3-D (Figure 2) is a C-arm-based technology which obtains images via 190-degree rotation around the patient prior to reformatting the data into a 3D anatomic map. Similar to the Airo Mobile system, after instrument registration via scanning camera, the reference 


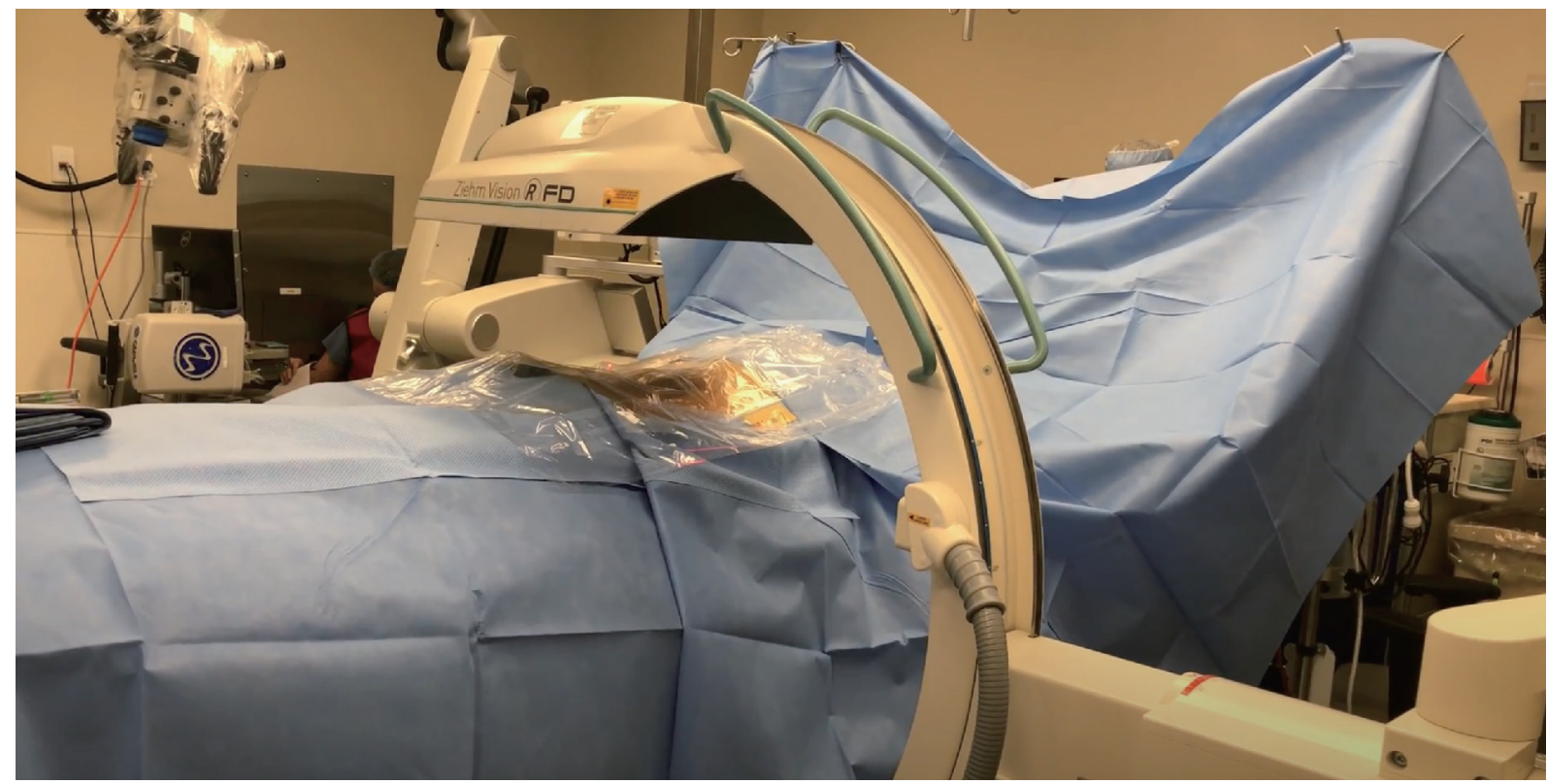

Figure 2 Ziehm Vision FD Vario 3D.
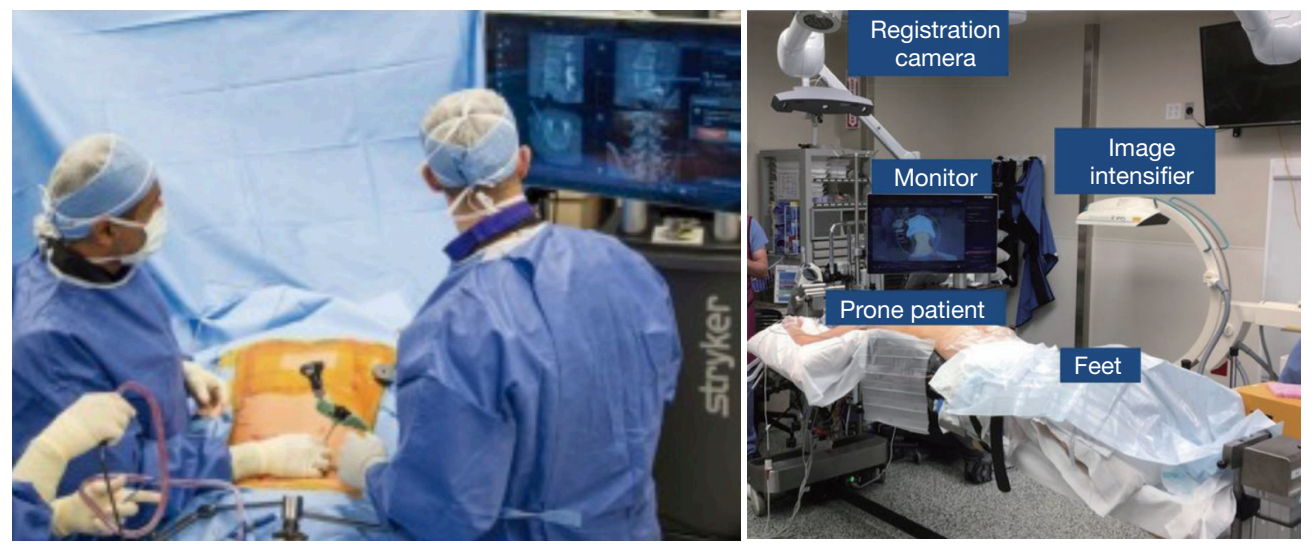

Figure 3 Stryker SpineMask.

clamps may not be touched, as any movement of the registered data points will lead to inaccurate mapping. If the registration loses accuracy, repeat scanning will be necessary to provide reliable stereotactic imaging (5).

In an effort to bypass some of the limitations of other systems as described above, the Stryker SpineMask Tracker offered a different form of referencing. The reference trackers in this system are applied directly onto the patient's skin to prevent obstacles, such as obstruction of camera by hand positioning, caused by reference trackers or movement of reference points after referencing (Figure 3). However in order for accurate real-time mapping the camera must have full view of 5 of the 31 LEDs that are actively tracking, which are placed surrounding the surgical field. Also, the operative field cannot exceed the predefined size parameters of the reference points. Deep retraction or undue skin tension can alter the reference point locations and the accuracy will be negatively impacted and thus this method of navigation is not typically utilized for large open surgery (5). The SpineMask is typically used for MIS surgery. If used for open surgery the SpineMask may be placed at an area distal or proximal to the surgical wound to prevent 
retraction of the reference points. With multiple accurate options available for navigation available many surgeons incorporated CAN into their practices.

\section{Operating room preparation/set up}

Operating room set-up is variable for the use of navigation based on surgeon preference, as well as navigation platform used. Some systems, such as the Airo Mobile, have the navigation system attached to the operating bed. Other systems utilize monitors at bedside and reference trackers attached to patient anatomic landmarks. Many articles discuss operating room setup, with common preferences that include a large operative suite with navigation and image intensifiers placed as per surgeon preference, and reference cameras at either the head or foot of the bed of the patient depending on location of the procedure $(4,5)$. For a typical MIS lumbar procedure, our surgeons will use the SpineMask laid over the thoracic spine (to decrease potential retraction/disruption the SpineMask), while both the monitor and the camera are placed at the foot of the bed for optimum viewing.

\section{Navigation effects on efficiency/safety}

A primary reason for the growth of navigation in spine surgery was the proposed increased safety and efficiency of pedicle screw placement as compared to freehand or fluoroscopy-guided procedures. Many studies discuss and affirm early findings regarding the improved safety with the use of computer assisted navigation (6-16). Amiot et al. demonstrated the difference in safety between free hand technique and CAN. The error rate was $15.3 \%$ for 544 screws from $\mathrm{T} 5$ to $\mathrm{S} 1$ when placed with freehand technique, while only $5.4 \%$ for the 294 screws inserted utilizing CAN (4).

Yu et al. evaluated the accuracy of 2,062 thoracic and lumbar pedicle screws utilizing intraoperative computer assisted navigation (17). Their results showed $4.6 \%$ of screws breached the pedicles $>2 \mathrm{~mm}$ when navigated placement was utilized, versus a $16 \%$ misplacement rate when using freehand technique $(\mathrm{P}<0.001)$. They also showed a significant lower mean time of pedicle screw implantation and operating time in the CAN group. This finding has been repeated in multiple other studies with Luther et al. finding $88 \%$ percent of navigated pedicle screws found to have no breach versus $82 \%$ of cases with lateral fluoroscopy $(\mathrm{P}<0.001)$, showing the superiority of the accuracy using navigation (18). A systematic review with 7,000 total pedicle screw placed by Shin et al. confirmed the earlier results, with overall incidence of pedicle screw perforation using CAN techniques to be $6 \%$ as compared to $15 \%$ for freehand technique (19). While pedicle screw perforation was significantly different, there was no significant difference in neurologic complications in the two groups. These findings echoed similar findings from an earlier meta-analysis by Verma et al. which showed no significant difference in neurological injury between navigated and non-navigated pedicle screw placement $(\mathrm{P}=0.07)(20)$. These studies affirmed the notion that navigated pedicle screw placement is more accurate than placement using freehand or fluoroscopy, however, the clinical significance has largely been deemed equivocal based on current studies. Other considerations for use of CAN is to determine the effect it has on screw diameter, screw/pedicle diameter ratio, and need for revision surgery. Luther et al. showed screws placed with CAN were significantly larger, with a significantly larger screw/pedicle diameter ratio, and significantly fewer revision procedures were performed (18). This evidence helps support the use of CAN for spine surgery.

\section{Radiation effects of navigation}

The widespread use of navigation in spine surgery was also propelled by the significant radiation exposure of both patient and surgeon using pre-navigation techniques. Theocharopoulos et al. showed that radiation exposure for a spine surgeon was 50 times the lifetime radiation dose of a hip surgeon, highlighting the need to limit radiation exposure (21). Multiple studies have shown decreased radiation exposure with the use of CAN as compared to fluoroscopy guided spine procedures (22-24). Specifically, Gebhard et al. found radiation doses were reduced from a median of 1,091 mGy using fluoroscopy (average fluoroscopy time of 40 seconds) for thoracolumbar instrumentation versus $432 \mathrm{mGy}$ in CT-based navigation techniques (25).

In 2000, Rampersaud et al. evaluated the surgeon radiation exposure during a traditional open posterior lumbar fusion in cadavers and showed that even if fluoroscopy time was limited to $2 \mathrm{~min}$ per case, the annual threshold for radiation exposure (measured in mrem) was surpassed after just 300 cases (26). Bindal et al. found that the radiation exposure to an MIS surgeon during a transforaminal lumbar interbody fusion surpassed the 
occupational exposure limit after just 194 cases (27). These studies furthered the notion that the amount of surgeon and patient radiation exposure necessitated new imaging technology for safe practice.

One of the main benefits of CAN was reducing radiation exposure to the surgeon. Kim et al. performed one of the initial studies evaluating the role of navigation as a means to reduce radiation exposure and showed that navigation significantly reduced fluoroscopy time by 90 seconds per case, and completely negated the radiation exposure to the surgeon, who could leave the room during fluoroscopy (28). In addition to reduced radiation exposure to the surgeon, Kraus et al. showed that navigation also decreased patient exposure during open spine surgery. In their cohort of 40 patients undergoing posterior lumbar fusion, their study found an average effective dose of $0.4 \mathrm{mGy}$ in the computer assisted navigation group as compared to $5.03 \mathrm{mGy}$ experienced in the fluoroscopy group (24). These studies showed the superiority of CAN in regards to radiation exposure for both the patient and surgeon.

\section{Navigation for MIS}

The use of computer assisted navigation has allowed for the field of MIS surgery to expand considerably. One initial problem was concern for increased patient radiation exposure as compared to fluoroscopic guided procedures, however, the use of CAN was found to reduce radiation exposure by more than $90 \%$ compared to traditional fluoroscopic guided percutaneous surgical techniques (29). As the surgeon leaves the room or stands behind a lead shield during the scanning component, the radiation risks are mitigated during navigated procedures. The accuracy of registration has improved as the technology has improved, allowing for reliable, real-time feedback regarding relative positions of instruments within the three-dimensional map provided by intra-operative imaging (5). This accuracy and reliability of computer generated anatomy is important when exposure is limited in MIS surgery.

Virk et al. discussed the benefits of navigation usage in MIS, particularly with the complex anatomy associated with placing C1-C2 instrumentation (30). The ability for the navigation systems to provide accurate real-time feedback is reliant on reference markers which vary from system to system. These markers are generally attached to anatomical structures within or close to surgical field. Care must be taken to prevent any movement of attached trackers after scanning is complete to maintain reliable navigation feedback, but this can be difficult in certain procedures secondary to limited space and relatively bulky reference markers (29). One skin based marking system used for MIS, TLIF was discussed by Vaishnav et al. as a means to prevent surgical field overcrowding. Their study demonstrated the efficacy of a less bulky registration system that could be used for cases involving smaller incisions. Their navigation cohort resulted in significantly shorter operative times (median 92 vs. 108 minutes, $\mathrm{P}<0.0001$ ) and less blood loss (median 25 vs. $50 \mathrm{~mL}, \mathrm{P}=0.007$ ), as compared to the fluoroscopy group (31). And while their navigation group required additional time for image acquisition, the total time (image acquisition + surgical procedure) was only slightly greater than that of procedures using fluoroscopy alone (median 113 vs. 108 mins). The median set up time using the skin tracking system was 23 minutes, with over $70 \%$ of cases being set-up in $<25$ minutes, demonstrating that the preoperative set up was not laborious. Similar to studies above, they observed a $29 \%$ reduction in the median radiation dose to the patient as compared to fluoroscopic procedures (31).

\section{Implementation of navigation}

As the current medical care system gravitates towards the most cost-effective possible system, the upfront cost of navigation was originally seen as prohibitive in some cases. More recent data has shown a reduced number of re-operations with use of navigation, however, leading to the widespread adoption of its use (5). Zausinger et al. showed an average cost savings of $\$ 27,813.18$ by avoiding a revision procedure (32). Al-Khouja et all performed a costeffectiveness analysis and showed the cost of intra-operative imaging to range from $\$ 8820.51$ to $\$ 9188.87$ and a cost range of revision surgery to be $\$ 17,650$ to $\$ 39,643$, further illustrating the cost savings of avoiding revision surgery despite a higher upfront cost for CAN (33). As efficacy, safety, and cost effectiveness have seemingly been proven at this juncture, it is likely that the use of navigation will only continue to increase.

\section{Navigation for tumors}

The initial discussion regarding the use of stereotactics for tumor removal was seen in the neurosurgical skull-based literature in the 1980s (34). Arand et al. used this technology to resect a small group of thoracic tumors in a 2002 study, showing the safety and efficacy of the use of navigation for 
tumor resection (35). Van Royen et al. demonstrated similar findings in a study reporting resection of osteoid osteomas of the thoracic and lumbar spine in 5 patients (36). They showed that computer assisted 3D navigation can be a safe means of guided excision with a high-speed burr, though no comparative data was given in regards to tumor excision with previous imaging modalities. All of the patients in their study showed complete eradication of their lesions without intra-operative complications and without recurrence up to 3 years follow-up.

Smitherman et al. discussed the use of stereotaxy for hemicorpectomy resection of a large tumor, describing a new use for computer assisted navigation (37). This study showed a safe means of resecting a thoracic tumor with clear margins using real time navigation. Ando et al. provided evidence of effective navigation application in primary bone tumor resection (38). In total, 18 primary bone tumors were resected and showed no intraoperative complications related to the navigation procedure or equipment, no screw misplacement, mechanical implant failure, or local recurrence in any patients. While further studies are required to elucidate the benefit of navigation $v$. current standard of care in spine tumor resection, current available literature supports its safety and accuracy, and may lead to an expansion in its use.

\section{Future directions of navigation}

With the growth of navigation, innovation is paramount to improve safety and efficiency. Improved monitors for visualization, optimized operating suites for navigation, smaller registration trackers are all areas that are receiving current attention. One attempted solution was the Airo Mobile Brainlab $^{\odot}$ which allowed for a custom OR table with 360 degrees of rotation, though future outcomes studies are required to see if these innovations provide tangible benefit in outcomes. It is likely that platforms will continue to arise in a similar manner to optimize the use of navigation. Another innovation that will continue to be implemented is the use of augmented reality headsets with navigation in real time and directly overlaid on the patient, instead of a screen. Once this technology becomes more end user friendly its implementation will increase significantly in our opinion.

Continuing to make these platforms more cost-effective is another future direction. As the platform becomes more affordable, computer assisted navigation utilization will likely increase. Studies show decreased operative time with pedicle screw placement using navigation as compared to fluoroscopy though surgeon familiarity with navigation may be a contributing factor (16,33). Expanding applications of the same technology will grow navigation-based technology and use. CT-MRI co-registration is another feature that can enhance CAN application in patients presenting with soft tissue masses without bony involvement. Preoperative MRI and CT could be used for registration allowing for decreased intra-operative radiation and likely less set up time. Robotic assisted surgery is another current expansion opportunity for navigation, though its cost may be prohibitive in some situations.

\section{Conclusions}

The growth of navigation technology in spine surgery is due to the improved safety and efficacy of this platform. As more literature becomes available showing its benefits, the role of navigation will likely expand in spine surgery applications. Further innovation is necessary to improve performance and show cost-effectiveness compared to alternative technologies. The use of computer assisted navigation has become more prevalent in spine surgery, and its impact is likely to continue to increase over time.

\section{Acknowledgments}

Funding: None.

\section{Footnotes}

Provenance and Peer Review: This article was commissioned by the Guest Editor (Dr. Sheeraz Qureshi) for the series "Current State of Intraoperative Imaging" published in Annals of Translational Medicine. The article was sent for external peer review organized by the Guest Editor and the editorial office.

Conflicts of Interest: All authors have completed the ICMJE uniform disclosure form (available at http://dx.doi. org/10.21037/atm-20-1335). The series "Current State of Intraoperative Imaging" was commissioned by the editorial office without any funding or sponsorship. HSS reports other from Amedica, other from Biorestorative Therapies, other from Paradigm Spine, other from Prosydian Medical, other from Providence Medical Technology, other from Spinewave, outside the submitted work. The authors have no other conflicts of interest to declare. 
Ethical Statement: The authors are accountable for all aspects of the work in ensuring that questions related to the accuracy or integrity of any part of the work are appropriately investigated and resolved.

Open Access Statement: This is an Open Access article distributed in accordance with the Creative Commons Attribution-NonCommercial-NoDerivs 4.0 International License (CC BY-NC-ND 4.0), which permits the noncommercial replication and distribution of the article with the strict proviso that no changes or edits are made and the original work is properly cited (including links to both the formal publication through the relevant DOI and the license). See: https://creativecommons.org/licenses/by-nc-nd/4.0/.

\section{References}

1. Nolte LP, Visarius H, Arm E, et al. Computer-aided fixation of spinal implants. J Image Guid Surg 1995;1:88-93.

2. Lonjon N, Chan-Seng E, Costalat V, et al. Robot-assisted spine surgery: feasibility study through a prospective casematched analysis. Eur Spine J 2016;25:947-55.

3. Lavallée S, Sautot P, Troccaz J, et al. Computer-assisted spine surgery: a technique for accurate transpedicular screw fixation using CT data and a 3-D optical localizer. J Image Guid Surg 1995;1:65-73.

4. Amiot LP, Lang K, Putzier M, et al. Comparative results between conventional and computer-assisted pedicle screw installation in the thoracic, lumbar, and sacral spine. Spine 2000;25:606-14.

5. Overley SC, Cho SK, Mehta AI, et al. Navigation and Robotics in Spinal Surgery: Where Are We Now? Neurosurgery 2017;80:S86-99.

6. Ishikawa Y, Kanemura T, Yoshina G, et al. Clinical accuracy of three-dimensional fluoroscopybased computerassisted cervical pedicle screw placement: a retrospective comparative study of conventional versus computerassisted cervical pedicle screw placement. Clinical article. J Neurosurg Spine 2010;13:606-11.

7. Ito H, Neo M, Yoshida M, et al. Efficacy of computerassisted pedicle screw insertion for cervical instability in RA patients. Rheumatol Int 2007;27:567-74.

8. Kotani $Y$, Abumi K, Ito $M$, et al. Improved accuracy of computer-assisted cervical pedicle screw insertion. J Neurosurg 2003;99:257-63.

9. Kotani Y, Abumi K, Ito M, et al. Accuracy analysis of pedicle screw placement in posterior scoliosis surgery: comparison between conventional fluoroscopic and computer-assisted technique. Spine 2007;32:1543-50.

10. Laine T, Lund T, Ylikoski M, et al. Accuracy of pedicle screw insertion with and without computer assistance: a randomised controlled clinical study in 100 consecutive patients. Eur Spine J 2000;9:235-40.

11. Lee GY, Massicotte EM, Rampersaud YR. Clinical accuracy of cervicothoracic pedicle screw placement: a comparison of the "open" lamino-foraminotomy and computer-assisted techniques. J Spinal Disord Tech 2007;20:25-32.

12. Liu YJ, Tian W, Liu B, et al. Accuracy of CT-based navigation of pedicle screws implantation in the cervical spine compared with X-ray fluoroscopy technique. Zhonghua Wai Ke Za Zhi 2005;43:1328-30.

13. Merloz P, Troccaz J, Vouaillat H, et al. Fluoroscopy-based navigation system in spine surgery. Proc Inst Mech Eng $\mathrm{H}$ 2007;221:813-20.

14. Nakashima H, Sato K, Ando T, et al. Comparison of the percutaneous screw placement precision of isocentric C-arm 3-dimensional fluoroscopy-navigated pedicle screw implantation and conventional fluoroscopy method with minimally invasive surgery. J Spinal Disord Tech 2009;22:468-72.

15. Rajasekaran S, Vidyadhara S, Ramesh P, et al. Randomized clinical study to compare the accuracy of navigated and non-navigated thoracic pedicle screws in deformity correction surgeries. Spine 2007;32:E56-64.

16. Richter M, Cakir B, Schmidt R. Cervical pedicle screws: conventional versus computer-assisted placement of cannulated screws. Spine 2005;30:2280-7.

17. Yu X, Xu L, Bi LY. Spinal navigation with intra-operative $3 \mathrm{D}$ imaging modality in lumbar pedicle screw fixation. Zhonghua Yi Xue Za Zhi 2008;88:1905-8.

18. Luther N, Iorgulescu JB, Geannette C, et al. Comparison of navigated versus non-navigated pedicle screw placement in 260 patients and 1434 screws: screw accuracy, screw size, and the complexity of surgery. J Spinal Disord Tech 2015;28:E298-303.

19. Shin BJ, James AR, Njoku IU, et al. Pedicle screw navigation: a systematic review and meta-analysis of perforation risk for computer-navigated versus freehand insertion. J Neurosurg Spine 2012;17:113-22.

20. Verma R, Krishan S, Haendlmayer, K, et al. Functional outcome of computer-assisted spinal pedicle screw placement: a systematic review and meta-analysis of 23 studies including 5,992 pedicle screws. Eur Spine J 2010;19:370-5. 
21. Theocharopoulos N, Perisinakis K, Damilakis J, et al. Occupational exposure from common fluoroscopic projections used in orthopaedic surgery. J Bone Joint Surg Am 2003;85:1698-703.

22. Gebhard F, Kraus M, Schneider E, et al. Radiation dosage in orthopedics: a comparison of computer-assisted procedures. Unfallchirurg 2003;106:492-7.

23. Gebhard F, Weidner A, Liener UC, et al. Navigation at the spine. Injury 2004;35:S-A35-45.

24. Kraus MD, Krischak G, Keppler P, et al. Can Computerassisted Surgery Reduce the Effective Dose for Spinal Fusion and Sacroiliac Screw Insertion? Clin Orthop Relat Res 2010;468:2419-29.

25. Gebhard FT, Kraus MD, Schneider E, et al. Does computer-assisted spine surgery reduce intraoperative radiation doses? Spine (Phila Pa 1976) 2006;31:2024-7; discussion 2028.

26. Rampersaud YR, Foley KT, Shen AC, et al. Radiation exposure to the spine surgeon during fluoroscopically assisted pedicle screw insertion. Spine 2000;25:2637-45.

27. Bindal RK, Glaze S, Ognoskie M, et al. Surgeon and patient radiation exposure in minimally invasive transforaminal lumbar interbody fusion. J Neurosurg Spine 2008;9:570-3.

28. Kim CW. Use of navigation-assisted fluoroscopy to decrease radiation exposure during minimally invasive spine surgery. Spine J 2008;8:584-90.

29. Vaishnav AS, Othman YA, Virk SS, et al. Current state of minimally invasive spine surgery. J Spine Surg 2019;5:S2-10.

30. Virk S, Qureshi S. Navigation in minimally invasive spine surgery. J Spine Surg 2019;5:S25-30.

Cite this article as: Rawicki N, Dowdell JE, Sandhu HS. Current state of navigation in spine surgery. Ann Transl Med 2021;9(1):85. doi: 10.21037/atm-20-1335
31. Vaishnav AS, Merrill R, Sandhu H, et al. A Review of Techniques, Time-demand, Radiation Exposure and Outcomes of Skin-anchored Intra-operative 3D Navigation in Minimally Invasive Lumbar Spinal Surgery. Spine (Phila Pa 1976) 2020;45:E465-76.

32. Zausinger S, Scheder B, Uhl E, et al. Intraoperative computed tomography with integrated navigation system in spinal stabilizations. Spine (Phila $\mathrm{Pa} 1976$ ) 2009;34:2919-26.

33. Al-Khouja L, Shweikeh F, Pashman R, et al. Economics of image guidance and navigation in spine surgery. Surg Neurol Int 2015;6:S323-6.

34. Roberts DW, Strohbehn JW, Hatch JF, et al. A frameless stereotaxic integration of computerized tomographic imaging and the operating microscope. J Neurosurg 1986;65:545-9.

35. Arand M, Hartwig E, Kinzl L, et al. Spinal navigation in tumor surgery of the thoracic spine: first clinical results. Clin Orthop Relat Res 2002;(399):211-8.

36. Van Royen BJ, Baayen JC, Pijpers R, et al. Osteoid osteoma of the spine: a novel technique using combined computer-assisted and gamma probe-guided highspeed intralesional drill excision. Spine (Phila Pa 1976) 2005;30:369-73.

37. Smitherman SM, Tatsui CE, Rao G, et al. Image-guided multilevel vertebral osteotomies for en bloc resection of giant cell tumor of the thoracic spine: case report and description of operative technique. Eur Spine J 2010;19:1021-8.

38. Ando K, Kobayashi K, Machino M, et al. Computed tomography-based navigation system-assisted surgery for primary spine tumor. J Clin Neurosci 2019;63:22-6. 